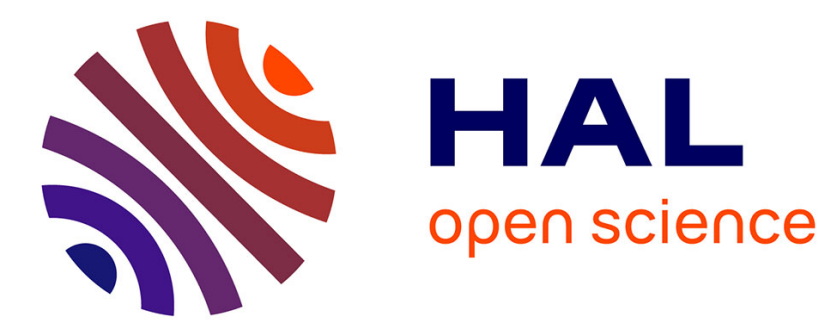

\title{
A Rapid Decrease of the Hydrogen Corona of Mars
}

John T. Clarke, Jean-Loup Bertaux, Jean-Yves Chaufray, G. Randall

Gladstone, Eric Quémerais, J. K. Wilson, Dolon Bhattacharyya

\section{To cite this version:}

John T. Clarke, Jean-Loup Bertaux, Jean-Yves Chaufray, G. Randall Gladstone, Eric Quémerais, et al.. A Rapid Decrease of the Hydrogen Corona of Mars. Geophysical Research Letters, 2014, 41 (22), pp.8013-8020. 10.1002/2014GL061803 . hal-01082647

\section{HAL Id: hal-01082647 https://hal.science/hal-01082647}

Submitted on 19 Jul 2020

HAL is a multi-disciplinary open access archive for the deposit and dissemination of scientific research documents, whether they are published or not. The documents may come from teaching and research institutions in France or abroad, or from public or private research centers.
L'archive ouverte pluridisciplinaire HAL, est destinée au dépôt et à la diffusion de documents scientifiques de niveau recherche, publiés ou non, émanant des établissements d'enseignement et de recherche français ou étrangers, des laboratoires publics ou privés. 


\section{Geophysical Research Letters}

\section{RESEARCH LETTER}

10.1002/2014GL061803

Key Points:

- A strong variation in escape of

hydrogen from the martian

atmosphere is reported

- The atmospheric escape from Mars has a strong seasonal dependence

- Atmospheric escape from Mars is strongly enhanced during a global dust storm

Correspondence to:

J. T. Clarke,

jclarke@bu.edu

\section{Citation:}

Clarke, J. T., J.-L. Bertaux, J.-Y. Chaufray, G. R. Gladstone, E. Quemerais, J. K. Wilson, and D. Bhattacharyya (2014), A rapid decrease of the hydrogen corona of Mars, Geophys. Res. Lett., 41, 8013-8020, doi:10.1002/ 2014 GL061803.

Received 11 SEP 2014 Accepted 31 OCT 2014 Accepted article online 6 NOV 2014 Published online 28 NOV 2014

\section{A rapid decrease of the hydrogen corona of Mars}

\author{
J. T. Clarke' , J.-L. Bertaux ${ }^{1,2}$, J.-Y. Chaufray ${ }^{2}$, G. R. Gladstone ${ }^{3}$, E. Quemerais ${ }^{2}$, J. K. Wilson ${ }^{4}$, \\ and D. Bhattacharyya ${ }^{1}$
}

${ }^{1}$ Center for Space Physics, Boston University, Boston, Massachusetts, USA, ${ }^{2}$ LATMOS/CNRS, Guyancourt, France, ${ }^{3}$ Southwest Research Institute, San Antonio, Texas, USA, ${ }^{4}$ U. New Hampshire, Durham, New Hampshire, USA

\section{Introduction}

The upper atmospheres of all three terrestrial planets are slowly evaporating into space [Jakosky and Phillips, 2001], with the highest loss rate for the lightest atom, hydrogen. At Venus, Mars, and the Earth water vapor is trapped close to the surface by condensation due to the decreasing temperature with altitude. Solar near-UV radiation penetrates sufficiently deep into the atmospheres to dissociate $\mathrm{H}_{2} \mathrm{O}$, and the resulting $\mathrm{H}$ and $\mathrm{O}$ atoms then diffuse into the upper atmosphere. Both $\mathrm{H}$ and $\mathrm{O}$ atoms may be lost into space, $\mathrm{H}$ by both Jeans' escape and nonthermal processes, and the heavier O mainly by nonthermal processes [Hunten, 1982; Fox, 2014]. There are several lines of evidence for the loss of large amounts of water by this process over geologic time, including the elevated ratios of atmospheric D/H compared with terrestrial Standard Mean Ocean Water (SMOW) [Owen, 1992]. From the higher evaporation rate of $\mathrm{H}$ compared with $\mathrm{D}$ due to the atomic mass difference, the present elevated $\mathrm{D} / \mathrm{H}$ ratios on Mars and Venus can be related by models to the total amount of water that has been lost into space [Yung and Kass, 1998; Bertaux et al., 1993; Krasnopolsky et al., 1998; Bertaux and Montmessin, 2001]. By contrast, in the Earth's atmosphere the $\mathrm{H}$ is replenished by ocean water, and the D/H ratio of SMOW is likely unmodified by escape [Paxton and Anderson, 1992]. In the case of Mars, with its weak gravity, a relatively large amount of gas is believed to have been lost over the last 4 billion years, corresponding to a potentially substantial initial surface depth of water. The present-day total rate of atmospheric escape from Mars is poorly understood, and there are large uncertainties in the relative importance and efficiency of the various processes [Chassefière and LeBlanc, 2004]. Understanding the details of escape today is a requirement to be able to extrapolate into the past to learn the history of martian water.

The main processes for atmospheric evaporation from the top of the martian atmosphere are thermal or Jeans' escape, non-thermal escape from photochemical processes, ion escape and outflow, and ion sputtering. Photochemical processes refer to dissociative recombination of molecular ions leading to fast fragment atoms, an important process for $\mathrm{O}$ and possibly $\mathrm{N}$ and $\mathrm{C}$. Ion escape refers to the pickup of newly created high altitude ions by the solar wind magnetic field, and outflow is a similar process in which bulk ion flows are accelerated by the solar wind convection electric field. lon sputtering results when pickup ions re-impact the atmosphere with enough energy to sputter off neutrals, which can then escape.

Observations of the martian Ly $\alpha$ emission by Mariners 6, 7, and 9 were analyzed in detail [Anderson and Hord, 1971; Anderson, 1974], giving number densities of $\mathrm{H}$ with altitude and an upward flux of $2 \times 10^{8} \mathrm{~cm}^{-2} \mathrm{~s}^{-1}$. The widely accepted framework for these processes is that $\mathrm{H}$ begins as water sublimated from the surface, then is photodissociated by near-UV sunlight and recombines into $\mathrm{H}_{2}$ [Hunten and McElroy, 1970; Hunten, 1982]. The $\mathrm{H}_{2}$ diffuses into the upper atmosphere where it can return to atomic form through chemical reactions with the martian ionosphere, and the resulting $\mathrm{H}$ atoms then diffuse up to the exobase. Fast $\mathrm{H}$ 

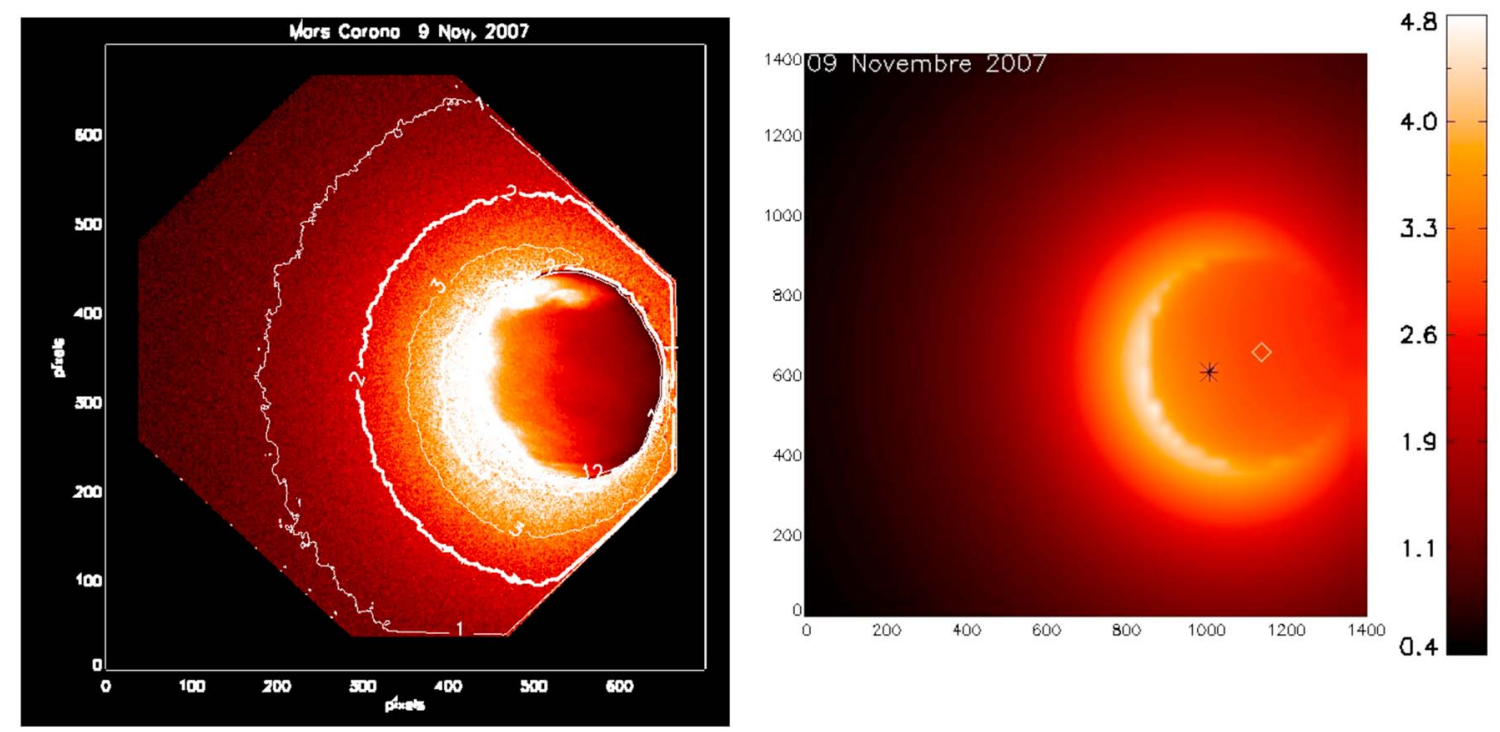

Figure 1. (left) Background-subtracted Hubble Space Telescope (HST) UV image of H Ly a emission from Mars with a band pass 115-140 nm on 9 November 2007. The pointing was offset to detect the more distant martian coronal emission profile, north is up and the Sun is toward the lower left. Contours at 1 kilo-Rayleigh brightness intervals are over-plotted. The disc of Mars appears noisy due to the high level of reflected UV sunlight from the surface and has been replaced by a far-UV image scaled down in intensity. The general shape of the coronal emission, but not the intensity level, repeats from day to day. (Right) Simulated image based on the radiative transfer code of Chaufray and Gladstone with intensity bar in kilo-Rayleighs. The model assumes spherical symmetry and includes the shadow of Mars, although photons are scattered into the shadow cone.

atoms result from dissociation of $\mathrm{H}_{2}$ by solar photons above the threshold energy, and the importance of another source of fast $\mathrm{H}$ atoms in reactions of the ions $\mathrm{CO}_{2}{ }^{+}$and $\mathrm{HCO}^{+}$has been pointed out [Krasnopolsky, 2002]. For any reaction that occurs well below the exobase, the $\mathrm{H}$ atom can be thermalized by collisions before reaching the exobase, providing the thermal component that dominates at lower altitudes of the exosphere. Exothermal reactions that occur near the exobase are sources of "hot" atoms, populating the external exosphere with many on escape trajectories. The SPICAM experiment on Mars Express has studied the martian $\mathrm{H}$ Ly $\alpha$ emission from its orbit within the exosphere, and found evidence for such a superthermal component of $\mathrm{H}$ atoms with $\mathrm{T}>500 \mathrm{~K}$ [Chaufray et al., 2008]. Recent observations from the Rosetta spacecraft have found profiles of hydrogen similar to prior observations, and also evidence for hot oxygen atoms in the extended atmosphere [Feldman et al., 2011]. The number and temperature of hot $\mathrm{H}$ can be directly related to the escape flux of $\mathrm{H}$ atoms and thereby water.

\section{Observations}

HST images with the ACS SBC camera of the martian ultraviolet $\mathrm{H}$ Ly $\alpha$ emission were obtained in three observing visits near solar minimum in Fall 2007 to investigate the nature and potential variability of the hydrogen corona ( $L_{s}=330-345$, southern summer and dust storm season). A follow-up observation was conducted in May 2014 near solar maximum when Mars was further from the Sun $\left(L_{S}=138\right.$, northern summer). We organized this observing campaign along with coordinated observations from the Mars Express SPICAM instrument [Bertaux et al., 2000], which observed the same H Ly $\alpha$ emission from within the corona over a period of several months but at a lower signal to noise ratio. The image shown in Figure 1 is the scaled difference between clear and filtered images (band pass $115-140 \mathrm{~nm}$ ) to remove the reflected solar continuum from the disc of Mars, and above an altitude of $\sim 0.2$ martian radii the emission is purely $\mathrm{H} \mathrm{Ly} \alpha$. A time series of images of the sky background emission was obtained close in time for an accurate subtraction, which is critical to determine the zero level far from the planet.

A theoretical model for the expected emission is also shown in Figure 1, based on a Chamberlain model for an assumed symmetric exosphere [Chamberlain, 1977] and taking into account multiple scattering of photons and the martian shadow. The radiative transfer model is a Monte Carlo radiative model first used to study the dayside venusian hydrogen corona [Chaufray et al., 2012] and adapted to Mars. This model was validated on Mars by comparison with results from the iterative matrix model used by Chaufray et al. to 


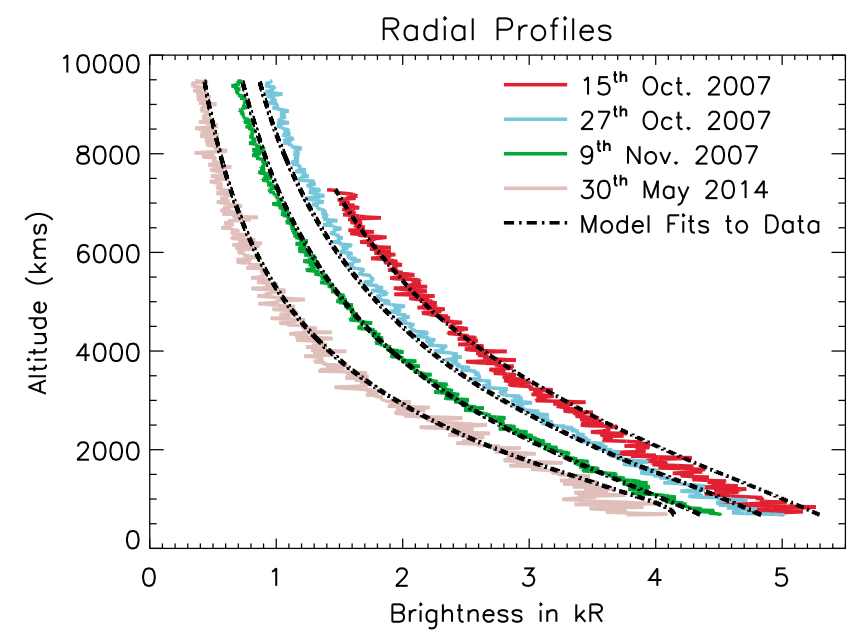

Figure 2. Comparison of intensity profiles of the $\mathrm{H}$ Ly $\alpha$ emission with distance on the 4 days of HST observations. The profiles are averaged over axial slices $+/-15^{\circ}$ from the sub-solar direction. The general shape of the profiles in the sub-solar direction is similar, but the level of emission steadily decreased with time, with the 2007 observations separated by 12 days in both intervals. The solar Ly $\alpha$ emission was constant over this time to within a few percent, so that does not explain the decrease. Parameters for the model fits (dot-dashed lines) are given in Table 1. Compared with more recent data on 30 May 2014, all of the 2007 emission levels were elevated. study the martian corona [Chaufray et al., 2008]. There are several clear differences seen in the HST images compared with the model. The vertical extent of the emission is greater than predicted by photochemical models or global circulation models for a low solar activity [Bougher et al., 2000; Krasnopolsky, 2002; Gonzalez-Galindo et al., 2009], which may reflect either a higher temperature or a superthermal component (no superthermal component is included in the models in this paper). In addition, there is a clear asymmetry with angle from the subsolar direction, with the emission brightness and vertical extent both decreasing as one moves azimuthally from the subsolar direction. This could imply a decreased source rate, and potentially changing thermal distribution, away from the subsolar point, but any specific conclusions will require further radiative transfer modeling.

The brightness of the sky background emission changes rapidly as HST orbits the Earth, with the changing line of sight through the geocorona. In the images of Mars, the coronal emission overfills the field of view, requiring a separate pointing far from Mars (offset by 5 arc minutes) to measure the background level. The data reported here include only the period of observations when the geocoronal background emission was less than $5 \mathrm{kR}$ to optimize the signal to noise in the faint coronal emission far from the planet. Another component of the sky background comes from solar photons scattered by interplanetary hydrogen, and this emission is Doppler-shifted by several Doppler widths from the martian line; thus, the martian atmosphere is optically thin to this emission above the limb, and it simply contributes to the uniform sky background.

Figure 2 shows plots comparing the observed altitude profiles on the three days of observations with those from the model, all in the subsolar direction for the sake of comparison. The intensity traces in Figure 2 begin at $1.2 \mathrm{R}_{\text {mars }}$ and extend out to the distance observed within the field of view on each day. Above an altitude of 1.2 $R_{\text {mars }}$ the other airglow emissions, notably the oxygen $130.4 \mathrm{~nm}$ line, have decreased to a negligible level (less than $20 \mathrm{R}$ ), and the wings of the imager point spread function from the bright disc emission are small compared with the Ly $\alpha$ emission. On DOY 288 the martian disc was centered in the field of view; thus, the field of view did not extend as far from the planet as on the other days, when the disc of Mars was offset toward one corner in the anti-solar direction.

\section{Data Analysis}

It is clear from the intensity traces in Figure 2 that the emission steadily decreased with time in Fall 2007, maintaining a shape generally consistent with a single $340 \mathrm{~K}$ hydrogen population but decreasing in intensity by more than $40 \%$ over 4 weeks' time. This trend and an initial discussion of its implications were first presented by Clarke et al. [2009]. The May 2014 data show an emission level lower than any of the 2007 data. Statistically better fits are obtained to the HST intensity profiles with a lower temperature plus a hot component, which implies a higher escape flux. Deriving accurate escape rates will require a detailed comparison with an exosphere/radiative transfer model, including non-spherical symmetry, and this work is ongoing. The model profiles correspond to $\mathrm{H}$ fluxes by Jean's escape of $2.6,2.1$, and $1.8 \times 10^{8} \mathrm{~cm}^{-2} \mathrm{~s}^{-1}$ in 2007 , and $6 \times 10^{7} \mathrm{~cm}^{-2} \mathrm{~s}^{-1}$ in 2014 [Chaufray et al., 2008]. Note that these are lower limits to the global escape flux, since they do not take into account nonthermal processes and possible superthermal atoms. The same decreasing trend in martian Ly $\alpha$ 
Table 1. Observations and Model Fits to the Profiles

\begin{tabular}{cccccc} 
& Day of Year & Temperature & $\begin{array}{c}\text { Exobase Density } \\
\left(\mathrm{cm}^{-3}\right)\end{array}$ & $\begin{array}{c}\text { Solar Lyman } \alpha \text { Flux }{ }^{\mathrm{a}} \\
\left(\mathrm{ph} \mathrm{cm}^{-2} \mathrm{~s}^{-1}\right)\end{array}$ & $\begin{array}{c}\text { Mars Solar } \\
\text { Longitude }\end{array}$ \\
\hline 2007: & 288 & $340 \mathrm{~K}$ & 50,000 & $1.33\left(\times 10^{11}\right)$ & $331^{\circ}$ \\
& 300 & $340 \mathrm{~K}$ & 40,000 & $1.29^{\prime \prime}$ & $337^{\circ}$ \\
& 313 & $340 \mathrm{~K}$ & 35,000 & $1.26^{\prime \prime}$ & $345^{\circ}$ \\
2014: & 150 & $260 \mathrm{~K}$ & 40,000 & $1.41^{\prime \prime}$ & $138^{\circ}$ \\
\hline
\end{tabular}

${ }^{\mathrm{a}}$ Solar Ly $\alpha$ flux at line center, data from the TIMED SEE instrument extrapolated to distance of Mars and corrected for solar rotation on the day of observation (http://lasp.colorado.edu/lisird/tss/composite_lyman_alpha.html). Uncertainties in values of density and temperature are discussed in the text and plotted in Figure 3.

emission was detected by SPICAM from within the atmosphere in coordinated observations [Chaffin et al., 2013]. Although these authors also describe a general trend of decreasing values with time, they reported a wide range of temperature and densities in their model fits (e.g., $T=200-1300 \mathrm{~K}$ and $n=1.5 \times 10^{4}-1.2 \times 10^{5} \mathrm{~cm}^{-3}$ ), a range of values that is not consistent with the HST observations at the same epoch. This may be related to the difficulty of interpreting observations with changing local times and illumination geometries from a limited range of altitude within the atmosphere in the SPICAM data.

Two separate radiative transfer models have been applied to HST observations of the martian hydrogen corona, one model from R. Gladstone developed originally for the Earth and Jupiter [Gladstone, 1988] and another based on an earlier geocoronal model from Chaufray et al. [2008]. The models gave similar results for the same assumed conditions, and in this paper we show the results from the Chaufray model. The model applies a Chamberlain model for the density of $\mathrm{H}$ atoms above the exobase, excluding satellite particles. This approach was verified by Monte Carlo modeling by Chen and Cloutier [2003], who found that this approach accounts best for exosphere changes due to charge exchange with solar wind protons. The hydrogen density below the exobase is derived from the resolution of the diffusion equation for atomic hydrogen through a $\mathrm{CO}_{2}$ atmosphere [Chaufray et al., 2008]. It assumes spherical symmetry in the distribution of the atmosphere and takes into account the direction of the incoming sunlight, the shadow of Mars, and the spectral shapes of the solar Ly $\alpha$ emission line and the martian line based on a maxwellian velocity distribution at an assumed temperature. The exosphere consists of a large number of $\mathrm{H}$ atoms following mainly ballistic trajectories, with a typical time of flight of a couple of hours. Over this flight time, only a small fraction of the atoms are removed by charge exchange with solar wind protons. The small fraction of the atoms with velocities that exceed the escape speed are lost into space, corresponding to a "boiling off" of the atmosphere while the great majority of atoms return to the exobase.

\section{Modeling}

A large number of model runs have been conducted, and the outputs have been compared with the radial profiles in Ly $\alpha$ brightness at various angles to the Mars-Sun line. The variables in the model are the $\mathrm{H}$ number density and temperature at the martian exobase, the presence of a superthermal component, and the solar Ly $\alpha$ flux at Mars (see Table 1). Several things have become clear from a comparison between the model outputs and the data:

1. there is a trade-off between the density and temperature values (Figure 3), in the sense that as one value decreases the other must be increased to fit the observed profiles-this arises from the broadening of the martian line with increasing temperature, which then scatters more of the solar line, so that the planet brightness increases with both increasing temperature and density

2. there is another trade-off between the bulk temperature and the inclusion of a superthermal component -the same observed profile can be fit almost equally well by a single temperature, or by a lower main temperature and an added small fraction superthermal component

3. altitude profiles at different angles to the Mars-Sun line result in different brightness distributions, implying that the corona may not be spherically symmetric-the significance of this will require more detailed radiative transfer modeling

4. the best fit temperature of $340 \mathrm{~K}$ in the 2007 data is higher than previously estimated for the exosphere near $400 \mathrm{~km}$ at this phase of the solar cycle [Forbes et al., 2008] —however, Krasnopolsky [2010] has pointed out difficulties in determining temperatures from spacecraft drag data at high altitudes 

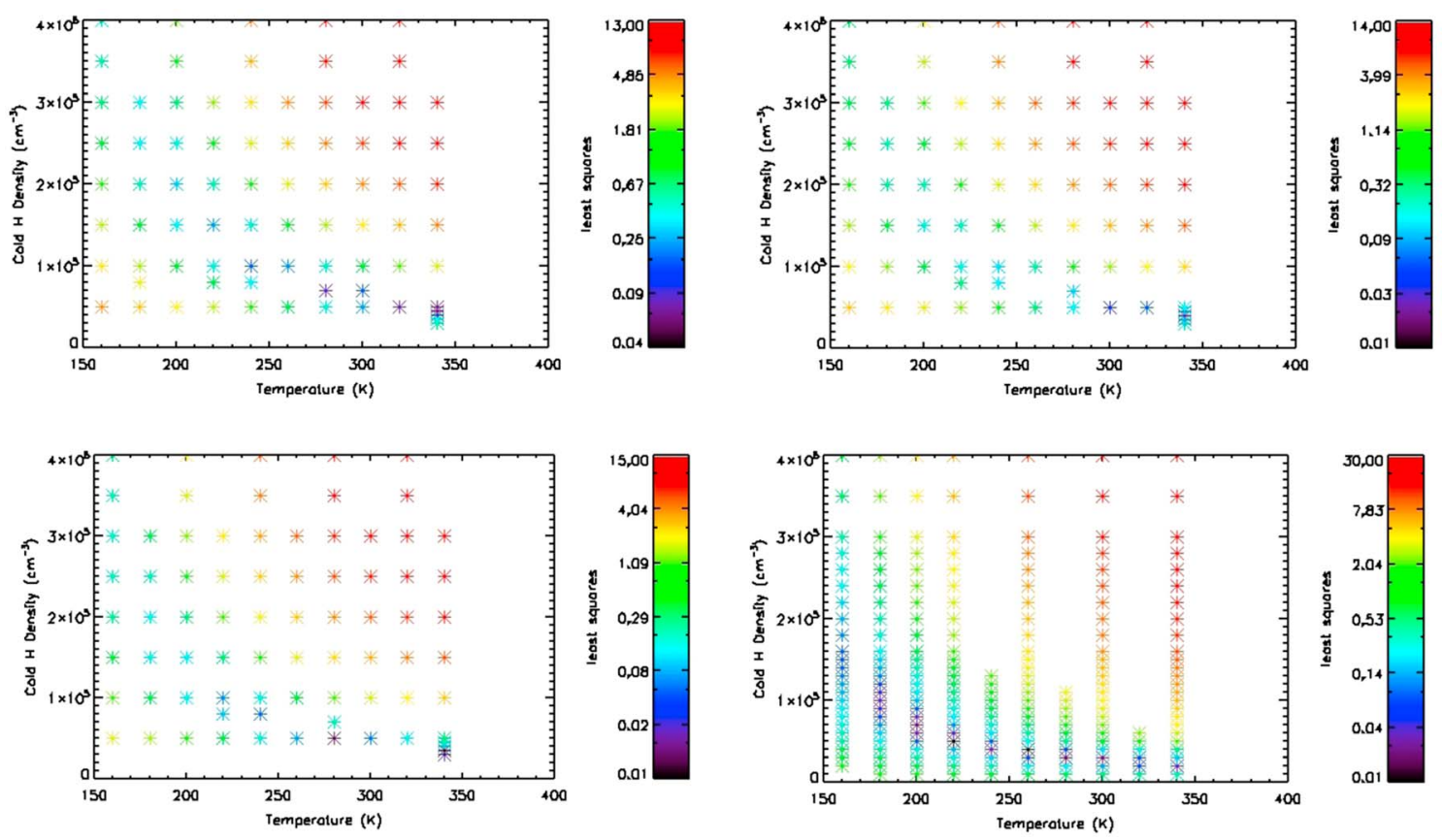

Figure 3. Plots showing the least squares fit chi-squared values for a range of assumed density and temperature values for each day of observations: (upper left) 15 October 2007, (upper right) 27 October 2007, (lower left) 9 November 2007, and (lower right) 30 May 2014. No hot atom population is included. On each day there is a range of values that give a good fit to the data, with a trade-off between values of density and temperature (see text).

We next present sample outputs from running a series of models to compare with the profiles on the four days of observations. Figure 3 shows that good fits to the observed emission profiles can be obtained with a range of density and temperature values; however, on each day the concentration of best fit values is for a relatively high temperature and low exobase density. It was not possible to obtain good fits on all days by varying the temperature alone, while it was possible to obtain good fits by varying the density alone. This is consistent with the knowledge that with a nearly constant solar UV flux and solar wind, the temperature of the bulk upper atmosphere is not expected to change. To compare similar quantities, we have therefore assumed a constant temperature and varied the density to obtain the best fit values for the 2007 data, while allowing both parameters to vary for the 2014 data near solar maximum.

\section{Interpretation}

The decreasing brightness of the extended emission might be explained by a decrease in the solar flux or an increased loss rate by interaction with the solar wind. During the 2007 observations near solar minimum, however, the solar UV flux did not change by more than a few percent, and there were no large changes in the quiet solar wind; thus, the observed decrease in coronal emission must have been caused by a variation within the atmosphere. The dissociation rate of $\mathrm{H}_{2}$ depends upon the UV solar flux, and its diffusion through the lower atmosphere takes longer than 4 weeks (see below). Another contributing factor that changes more rapidly is the sublimation rate and corresponding density of $\mathrm{H}_{2} \mathrm{O}$ in the lower atmosphere. In Fall 2007 Mars was moving away from the Sun (from solar longitude $L_{s}=330$ to $L_{s}=344$ during the HST observations), and the water vapor content of the gradually cooling lower atmosphere is known to decrease steadily at this epoch [Smith, 2009]. Maltagliati et al. [2011, 2013] have shown that the vertical extent of water vapor can exceed that predicted by martian climate models. In the SPICAM vertical water profiles in Mars year 29 during southern summer, the water mixing ratio was several times the model predictions. Water was observed at altitudes of 60-80 km with a value of 50-100 ppm in both hemispheres, and these values increased from $L_{s}=205$ to $L_{S}=270$. Super-saturation of water was also observed near $L_{S}=70-130$ in the cold season. When water vapor increases and moves to higher altitudes the rate of photodissociation by near-UV sunlight increases, providing an enhanced source of hydrogen for the upper atmosphere. Dust storms also occur in late martian summer, further heating the lower atmosphere and lifting the water to higher altitudes. The largest global dust storm 
over the prior 3 martian years occurred during summer 2007, and the dust opacity rapidly decreased during the HST observations [Smith, 2009]. Mars Express SPICAM/IR occultation measurements have been performed in Mars year 28 from $L_{S}=253^{\circ}$ to $300^{\circ}$. The data show that the $\mathrm{H}_{2} \mathrm{O}$ density up to $60-80 \mathrm{~km}$ increased significantly from $<20$ ppm before the dust storm to $50-100$ ppm after the dust storm in both hemispheres [Fedorova et al., 2006, 2008], and it has been shown by SPICAM data that the overall upper atmospheric density increases in this season [Forget et al., 2009]. By the process of elimination based on lack of changes in the solar UV flux, and the known seasonal changes and increase in water vapor in the middle atmosphere during and after the dust storm, the most likely factor changing the $\mathrm{H}$ density was the flux of hydrogen from the lower atmosphere. As the global climate cooled, the coronal hydrogen density decreased, leading to a rapid decrease in the escape of hydrogen from the martian atmosphere into space.

Estimates of the hydrogen number density, vertical fluxes, and diffusion rates are important considerations for this work to demonstrate that the proposed scenario for the upper atmosphere is reasonable. We present here rough estimates based on models for the structure and dynamics of the martian upper atmosphere to demonstrate the plausibility of the proposed interpretation of the data.

To achieve the derived escape fluxes, and have them decrease substantially over a few weeks, there must exist a column density of hydrogen atoms sufficient to supply the escape flux, and the vertical diffusion rate of $\mathrm{H}$ atoms through the mainly $\mathrm{CO}_{2}$ atmosphere must be sufficient to resupply the escaping flux. The vertical structure and diffusion of species within the martian atmosphere have been studied in comparison with results from a Mars global circulation (GCM) model [Forget et al., 1999]. For the sake of simplicity we consider the atmosphere separately in two layers, below $110 \mathrm{~km}$ where hydrogen diffuses upward either in the form of $\mathrm{H}_{2}$ or $\mathrm{H}_{2} \mathrm{O}$, and above $110 \mathrm{~km}$ where the $\mathrm{H}$ atoms diffuse to the exobase. W is the atmospheric vertical velocity at low altitudes and $W_{\text {diff }}$ the diffusion velocity at higher altitudes, taken from the GCM. The vertical diffusion time has first been estimated for a newly created $\mathrm{H}$ atom at $110 \mathrm{~km}$ up to the exobase. A rough estimate of the vertical transport time can be made using:

$$
\text { Time }=\int \frac{d Z}{W_{\text {diff }}}
$$

where $\mathrm{W}$ is the atmospheric vertical velocity at low altitudes, and the diffusion velocity at higher altitudes is taken from the GCM. This estimate is a lower limit, especially at low altitudes. The typical time found is 30-40 h with large differences from one latitude/longitude to another, and this order of magnitude estimated time is short compared with the separation of the HST observations. This implies that $\mathrm{H}$ atoms in the upper atmosphere are able to diffuse to the exobase in a time that is short compared with the observed changes in Ly $\alpha$ brightness.

A similar approach has been used to estimate the upward diffusion time for $\mathrm{H}_{2}$ from 10 to $110 \mathrm{~km}$ altitude. The time, $\int(d Z / W)$, is computed using the atmospheric vertical velocity $\mathrm{W}_{\text {atm }}$ from the GCM. At many positions (latitude, longitude) $W_{\text {atm }}$ is never greater than zero at all altitudes in this range. Choosing only the positions where $W_{a t m}>0$ results in a typical time of $\sim 50$ days (varying between 10 and 500 days), and the true value is likely longer since we ignored the locations where the atmosphere is stagnant. This is longer than the observed changes in Ly $\alpha$ brightness, consistent with the prevailing picture of a slow diffusion process resupplying hydrogen to the upper atmosphere. If, however, water is raised to high altitudes (50-80 km) and directly photo-dissociated by UV sunlight, this provides another source of atomic hydrogen in the upper atmosphere unconstrained by the diffusion of $\mathrm{H}_{2}$ from lower altitudes. These estimates suggest that while vertical diffusion of $\mathrm{H}_{2}$ might proceed slowly compared with the observed coronal decrease, the vertical diffusion of water under disturbed atmospheric conditions can be comparable to or shorter than the observed changes.

Another important consideration is to confirm that the decrease in $\mathrm{H}$ atom density is consistent with the number of atoms available and the derived Jeans escape fluxes. It is possible to estimate the flux needed to remove $40 \%$ of the hydrogen by:

$$
\text { Flux }_{\text {removed }}=0.4 \times \frac{N_{\text {atoms }}(H)}{\text { surface area } \times 4 \text { weeks }}
$$

where $\mathrm{N}_{\text {atoms }}(\mathrm{H})$ is an estimate of the number of hydrogen atoms in a column above $80 \mathrm{~km}\left(1.3 \times 10^{13} \mathrm{~cm}^{-2}\right)$. The flux removed is then $\sim 4.0 \times 10^{6}$ atoms $\mathrm{cm}^{-2} \mathrm{~s}^{-1}$, compared with a typical Jeans escape flux of 
$\sim 2 \times 10^{8}$ atoms $\mathrm{cm}^{-2} \mathrm{~s}^{-1}$. The proposed decrease in number density is thus well within the nominal escape flux. The vertical column of hydrogen above $80 \mathrm{~km}$ can be lost into space in $\sim 1$ day at the Jeans escape rate, a time comparable to the $30-40 \mathrm{~h}$ for diffusion of hydrogen through the upper atmosphere. This is consistent with the conclusion that the escape flux of $\mathrm{H}$ is limited by the source rate from the lower atmosphere.

In terms of the column of water in the lower atmosphere that is available to supply hydrogen to the upper atmosphere, this is large compared with the hydrogen column and escape flux in the upper atmosphere. The often-quoted 10 precipitable microns of water [Hunten and McElroy, 1970] in the atmosphere above the surface corresponds to a column of $5 \times 10^{19} \mathrm{~cm}^{-2} \mathrm{H}$ atoms, and for the derived $\mathrm{H}$ Jeans escape flux of $2 \times 10^{8} \mathrm{~cm}^{-2} \mathrm{~s}^{-1}$, the column would be lost in 10,000 years. This is long compared with the period of observations and the martian year, but short compared with the age of Mars. In practice the water in the atmosphere is in vapor pressure equilibrium with the surface, the ultimate source and reservoir of water for the atmosphere.

\section{Conclusions}

We have observed a rapid decrease in UV emission from the martian hydrogen corona, consistent with a corresponding decrease in $\mathrm{H}$ number density and escape flux. It appears likely that the water vapor content and altitude extent in the middle atmosphere are an important controlling factor of the density of the hydrogen corona and the escape flux. This was not anticipated and has important implications. It implies a variable escape flux of hydrogen and thereby water over the course of the martian year, and can be related to the historic rate of escape with orbital/seasonal changes on Mars. It remains for further observations to determine the relative importance of potential controlling factors. Does the variation in the corona occur with seasonal changes on Mars, or does it mainly occur during dust storms? In either case, historic martian climate changes will have had an important influence on the escape fluxes of water into space, significantly altering our understanding of the water history of Mars.

\section{Acknowledgments}

This work is based on observations with the NASA/ESA Hubble Space Telescope, obtained at the Space Telescope Science Institute (STScl), which is operated by AURA for NASA. These observations were supported by STScl grants GO-11170-01 and GO-12538-01 to Boston University. The authors thank Anna Fedorova for information about the Mars Express SPICAM/IR solar occultation data, and a helpful discussion about the altitude profiles of water in the middle atmosphere over time. All the HST data can be downloaded from the Space Telescope Science Institute at http://archive.stsci. edu/hst/search.php.

Andrew Dombard thanks three anonymous reviewers for their assistance in evaluating this paper.

\section{References}

Anderson, D. E. (1974), Mariner 6, 7, and 9 ultraviolet spectrometer experiment: Analysis of hydrogen lyman alpha data, J. Geophys. Res., 79, 1513-1518, doi:10.1029/JA079i010p01513.

Anderson, D. E., and C. W. Hord (1971), Mariner 6 and 7 ultraviolet spectrometer experiment: Analysis of hydrogen lyman-alpha data, J. Geophys. Res., 76, 6666-6673, doi:10.1029/JA076i028p06666.

Bertaux, J.-L., and F. Montmessin (2001), Isotopic fractionation through water vapor condensation: The deuteropause, a cold trap for deuterium in the atmosphere of Mars, J. Geophys. Res., 106(E12), 32,879-32,994, doi:10.1029/2000JE001358.

Bertaux, J.-L., J. T. Clarke, M. Mumma, T. Owen, and E. Quemerais (1993), A search for the deuterium lyman-alpha emission from the atmosphere of Mars, in "Science with the Hubble Space Telescope", ESO Proc. No. 44, 459-462.

Bertaux, J.-L., et al. (2000), The study of the martian atmosphere from top to bottom with SPICAM light on Mars Express, Planet. Space Sci., 48, 1303-1320, doi:10.1016/S0032-0633(00)00111-2.

Bougher, S. W., S. Engel, R. Roble, and B. Foster (2000), Comparative terrestrial planet thermospheres 3. Solar cycle variation of global structure and winds at solstice, J. Geophys. Res., 105, 17,669-17,692, doi:10.1029/1999JE001232.

Chaffin, M., J.-Y. Chaufray, I. Stewart, M. Montmessin, and N. Schneider (2013), Time variability of martian hydrogen escape, Geophys. Res. Lett., 41, 314-320, doi:10.1002/2013GL058578.

Chamberlain, J. W. (1977), Charge exchange in a planetary corona: Its effect on the distribution and escape of hydrogen, J. Geophys. Res., 82, 1-9, doi:10.1029/JA082i001p00001.

Chassefière, E., and F. LeBlanc (2004), Mars atmospheric escape and evolution; Interaction with the solar wind, Planet. Space Sci., 52, 1039-1058, doi:10.1016/j.pss.2004.07.002.

Chaufray, J.-Y., J.-L. Bertaux, F. Leblanc, and E. Quémerais (2008), Observation of the hydrogen corona with SPICAM on Mars express, Icarus, 195, 598-613, doi:10.1016/j.icarus.2008.01.009.

Chaufray, J.-Y., J.-L. Bertaux, E. Quémerais, E. Villard, and F. Leblanc (2012), Hydrogen density in the dayside venusian exosphere derived from lyman-alpha observations by SPICAV on Venus express, Icarus, 217, 767-778, doi:10.1016/j.icarus.2011.09.027.

Chen, Y., and P. A. Cloutier (2003), Martian hydrogen exosphere charge exchange with solar wind, J. Geophys. Res., 108(A10), 1381, doi:10.1029/2002JA009604.

Clarke, J. T., J.-L. Bertaux, J.-Y. Chaufray, G. R. Gladstone, E. Quémerais, and J. K. Wilson (2009), HST observations of the extended hydrogen corona of Mars, Bull. Am. Astr. Soc., 41, 49-11.

Fedorova, A., et al. (2006), Mars water vapor abundance from SPICAM IR spectrometer: Seasonal and geographic distributions, J. Geophys. Res., 111, 1-18, doi:10.1029/2006JE002695.

Fedorova, A., O. Korablev, J.-L. Bertaux, A. Rodin, F. Montmessin, and A. Reberac (2008), Vertical distributions of water vapor and aerosol in the martian atmosphere during the southern summer (MY28) by SPICAM-IR spectrometer on Mars-express, EPSC Abstracts, Vol. 3, EPSC2008-A-00559.

Feldman, P. D., et al. (2011), Rosetta-Alice observations of exospheric hydrogen and oxygen on Mars, Icarus, 214, 394-399, doi:10.1016/ j.icarus.2011.06.013.

Forbes, J., et al. (2008), Solar flux variability of Mars' exosphere densities and temperatures, Geophys. Res. Lett., 35, L01201, doi:10.1029/ 2007GL031904.

Forget, F., et al. (1999), Improved general circulation models of the martian atmosphere from the surface to above 80 km, J. Geophys. Res., 104, 24,155-24,175, doi:10.1029/1999JE001025. 
Forget, F., et al. (2009), Density and temperatures of the upper martian atmosphere measured by stellar occultations with Mars express SPICAM, J. Geophys. Res., 114, E01004, doi:10.1029/2009JE003086.

Fox, J. (2014), The escape of O from Mars: Sensitivity to the elastic cross sections, Icarus, 228, 375-385, doi:10.1016/j.icarus.2013.10.014.

Gladstone, G. R. (1988), UV resonance line dayglow emissions on Earth and Jupiter, J. Geophys. Res., 93, 14,623-14,630, doi:10.1029/ JA093iA12p14623.

Gonzalez-Galindo, F., F. Forget, M. López-Valverde, and M. Angelats (2009), A ground-to-exosphere martian general circulation model 2. Atmosphere during solstice conditions, J. Geophys. Res., 114, E04001, doi:10.1029/2008JE003277.

Hunten, D. M. (1982), Thermal and nonthermal escape mechanisms for terrestrial planets, Planet. Space Sci., 30, 773-783, doi:10.1016/00320633(82)90110-6.

Hunten, D. M., and M. B. McElroy (1970), Production and escape of hydrogen on Mars, J. Geophys. Res., 75, 5989-6001, doi:10.1029/ JA075i031p05989.

Jakosky, B., and R. Phillips (2001), Mars' volatile and climate history, Nature, 412, 237-244, doi:10.1038/35084184.

Krasnopolsky, V. A. (2002), Mars' upper atmosphere and ionosphere at low, medium, and high solar activities: Implications for evolution of water, J. Geophys. Res., 107(E12), 5128, doi:10.1029/2001JE001809.

Krasnopolsky, V. A. (2010), Solar activity variations of thermospheric temperatures on Mars and a problem of CO in the lower atmosphere, Icarus, 207, 638-647, doi:10.1016/j.icarus.2009.12.036.

Krasnopolsky, V., M. Mumma, and G. R. Gladstone (1998), Detection of atomic deuterium in the atmosphere of Mars, Science, 280, 1576-1580, doi:10.1126/science.280.5369.1576.

Maltagliati, L., et al. (2011), Evidence of water vapor in excess of saturation in the atmosphere of Mars, Science, 333, 1868-1871, doi:10.1126/ science. 1207957.

Maltagliati, L., et al. (2013), Annual survey of water vapor vertical distribution and water-aerosol coupling in the martian atmosphere observed by SPICAM/MEx solar occultations, Icarus, 223, 942-962, doi:10.1016/j.icarus.2012.12.012.

Owen, T. (1992), The composition and early history of the atmosphere of Mars, in Mars, edited by H. Keiffer et al., pp. 818-833, Univ. of Ariz. Press, Tucson.

Paxton, L. J., and D. E. Anderson (1992), Far ultraviolet remote sensing of Venus and Mars, in Venus and Mars: Atmospheres, lonospheres, and Solar Wind Interactions, Geophys. Monogr. Ser., vol. 113, edited by J. G. Luhmann et al., AGU, Washington, D. C., doi:10.1029/GM066p0113.

Smith, M. D. (2009), THEMIS observations of Mars aerosol optical depth from 2002-2008, Icarus, 202, 444-452, doi:10.1016/j. icarus.2009.03.027.

Yung, Y. L., and D. M. Kass (1998), Deuteronomy? A puzzle of deuterium and oxygen on Mars, Science, 280, 1545-1546, doi:10.1126/ science.280.5369.1545. 